\title{
Workshop on Using Emerging Parallel Architectures for Computational Science
}

\author{
Bertil Schmidt and Douglas Maskell \\ School of Computer Engineering, Nanyang Technological University, Singapore 639798 \\ \{asbschmidt, asdouglas\} @ntu.edu.sg
}

\begin{abstract}
The Workshop on Using Emerging Parallel Architectures for Computational Science, held in conjunction with ICCS 2009, provides a forum for exploring the capabilities of emerging parallel architectures such as GPUs, FPGAs, Cell B.E., and multi-cores to accelerate computational science applications.
\end{abstract}

Keywords: Computational Science, Parallel Computer Architectures, GPGPU, Reconfigurable Computing, Heterogeneous Multi-cores, High Performance Computing.

\section{Introduction to the Workshop}

Welcome to the Workshop on Using Emerging Parallel Architectures for Computational Science. This workshop has been motivated by the significant transformation of the computing landscape in recent years with the emergence of more powerful processing elements such as GPUs, FPGAs, Cell B.E., multi-cores, etc. On the multi-core front, Moore's Law has transcended beyond the single processor boundary with the prediction that the number of cores will double every 18 months. Going forward, the primary method of gaining processor performance will be through parallelism. Multi-core technology has visibly penetrated the global market. Accordingly to the Top500 lists, the HPC landscape has evolved from supercomputer systems into large clusters of dual or quad-core processors. Furthermore, GPUs, FPGAs and heterogeneous multi-cores have been shown to be formidable computing alternatives, where certain classes of applications witness more than one order of magnitude improvement over their GPP counterpart. Therefore, future computational science centers will employ resources such as FPGAs, GPUs and Cell architectures to serve as co-processors to offload appropriate compute intensive portions of applications from the servers. This workshop provides a forum for exploring the capabilities of emerging parallel architectures to accelerate computational science applications.

The technical program was put together by the Workshop Chairs Bertil Schmidt and Douglas Maskell and 19 members of a distinguished program committee. The workshop received 23 submissions. After an initial screening 21 submissions were reviewed by at least three experts in the field. Based on the reviews, 16 papers were selected for presentation at the workshop and inclusion in the workshop proceedings. 
We wish to thank the program committee members for submitting thoughtful reviews and all authors who submitted high-quality manuscripts. We plan to continue the workshop next year.

\section{Workshop Organizers}

Workshop Co-Chairs:

- $\quad$ Bertil Schmidt (Nanyang Technological University, Singapore)

- Douglas Maskell (Nanyang Technological University, Singapore)

Program Committee:

- Michael Huebner (University of Karlsruhe, Germany)

- Manfred Schimmler (University of Kiel, Germany)

- $\quad$ David Luebke (NVIDIA, USA)

- $\quad$ Simon See (SUN Microsystems)

- Neil Bergmann (University of Queensland, Australia)

- $\quad$ Philip Leong (Chinese University of Hong Kong, Hong Kong)

- Heiko Schroder (RMIT University, Australia)

- Alexandros Stamatakis (TU Munich, Germany)

- Dominique Lavenier (IRISA, France)

- Tarek El-Ghazawi (George Washington University, USA)

- Jaroslaw Zola (Iowa State University, USA)

- Michela Taufer (University of Delaware, USA)

- $\quad$ Rick Goh (IHPC, Singapore)

- $\quad$ Scott Emrich (University of Notre Dame, USA)

- Ananth Kalyanaraman (Washington State University, USA)

- $\quad$ Shi Haixiang (NTU, Singapore)

- $\quad$ Gerrit Voss (Fraunhofer IGD, Germany and NTU, Singapore)

- Weiguo Liu (NTU, Singapore)

- Malcolm Low (NTU, Singapore)

\section{List of Accepted Papers}

1. Solving Sparse Linear Systems on NVIDIA Tesla GPUs, M. Wang, H. Klie, M. Parashar, H. Sudan

2. Multi-walk Parallel Pattern Search Approach on a GPU Computing Platform, W. Zhu, J. Curry

3. Pairwise Distance Matrix Computation for Multiple Sequence Alignment on the Cell Broadband Engine, A. Wirawan, B. Schmidt, C.K. Kwoh

4. Evaluating the Jaccard-Tanimoto Index on Multi-Core Architectures, V. Sachdeva, D.M. Freimuth, C. Mueller

5. A particle-mesh integrator for galactic dynamics powered by GPGPUs, D. Aubert, M. Amini, R. David

6. Evaluation of the SUN UltraSparc T2+ Processor for Computational Science, M. Sandrieser, S. Pllana, S. Benkner 
7. Streamlining Offload Computing to High Performance Architectures, M. Purcell, O. Callanan, D. Gregg

8. Power Consumption of GPUs from a Software Perspective, C. Collange, D. Defour, A. Tisseran

9. A Note on Auto-tuning GEMM for GPUs, Y. Li, J. Dongarra, S. Tomov

10. Parallel calculating of the goal function in metaheuristics using GPU, W. Bozejko, C. Smutnicki, M. Uchronski

11. Fast Conjugate Gradients with Multiple GPUs, A. Cevahir, A. Nukada, S. Matsuoka

12. CUDA solutions for the SSSP problem, P.J. Martín, R. Torres, A. Gavilanes

13. Experiences With Mapping Non-Linear Memory Access Patterns into GPUs, E. Gutierrez, S. Romero, M.A. Trenas, O. Plata

14. A Massively Parallel Architecture for, Bioinformatics, G. Pfeiffer, S. Baumgart, J. Schroder, M. Schimmler

15. Accelerated Discovery of Discrete M-Clusters/Outliers on the Raster Plane using Graphical Processing Units, C. Trefftz, J. Szakas, I. Majdandzic, G. Wolffe

16. GPU accelerated RNA folding algorithm, G. Rizk, D. Lavenier 